\title{
Proposal of a new nomenclature for introns in protein-coding genes in fungal mitogenomes
}

\author{
Shu Zhang ${ }^{1}$ and Yong-Jie Zhang ${ }^{2^{*}}$ (D)
}

\begin{abstract}
Fungal mitochondrial genes are often invaded by group I or II introns, which represent an ideal marker for understanding fungal evolution. A standard nomenclature of mitochondrial introns is needed to avoid confusion when comparing different fungal mitogenomes. Currently, there has been a standard nomenclature for introns present in rRNA genes, but there is a lack of a standard nomenclature for introns present in protein-coding genes. In this study, we propose a new nomenclature system for introns in fungal mitochondrial protein-coding genes based on (1) three-letter abbreviation of host scientific name, (2) host gene name, (3), one capital letter $P$ (for group I introns), S (for group || introns), or U (for introns with unknown types), and (4) intron insertion site in the host gene according to the cyclosporin-producing fungus Tolypocladium inflatum. The suggested nomenclature was proved feasible by naming introns present in mitogenomes of 16 fungi of different phyla, including both basal and higher fungal lineages although minor adjustment of the nomenclature is needed to fit certain special conditions. The nomenclature also had the potential to name plant/protist/animal mitochondrial introns. We hope future studies follow the proposed nomenclature to ensure direct comparison across different studies.
\end{abstract}

Keywords: Nomenclature, Fungi, Mitogenome, Intron, Protein-coding gene

\section{INTRODUCTION}

Fungi constitute a huge group of highly diverse organisms, with 2.2-3.8 million estimated species and 144,000 currently known species on Earth (Hawksworth and Lücking 2017; Cannon et al. 2018). They were traditionally divided into four groups: chytridiomycetes, zygomycetes, ascomycetes, and basidiomycetes according to morphological traits associated with reproduction. Molecular phylogenetics and more recently phylogenomics recognized eight phyla in Fungi, namely Microsporidia, Cryptomycota, Blastocladiomycota, Chytridiomycota, Zoopagomycota, Mucoromycota, Ascomycota, and Basidiomycota (Spatafora et al. 2017). Aside from a few early divergent lineages and anaerobic organisms, almost all fungi contain mitochondria and mitogenomes in their cells (Bullerwell and Lang 2005; van der Giezen et al. 2005). Over recent years, mitogenomes of an increasing number of fungal species are sequenced. As of July 2019,

\footnotetext{
* Correspondence: zhangyj2008@sxu.edu.cn

${ }^{2}$ School of Life Science, Shanxi University, Taiyuan 030006, China Full list of author information is available at the end of the article
}

mitogenomes from at least 300 fungal species are available with representatives from all major fungal groups. Fungal mitogenomes typically contain 15 standard protein-coding genes, two rRNA genes and a variable number of tRNA genes. These protein-coding genes are atp6, atp8, atp9, cob, cox1, cox2, cox3, nad1, nad2, nad3, nad4, nad4L, nad5, nad6, and rps3 (Lang 2018), and some of them may be absent from certain fungal mitogenomes (Koszul et al. 2003).

Introns as mobile elements are frequently observed in mitochondrial protein-coding and/or rRNA genes of fungi. One gene may also be simultaneously invaded by multiple introns (e.g., four introns in $c o b$ and seven introns in cox 1 in Isaria cicadae) (Fan et al. 2019). Mitochondrial introns are divided into two groups (I and II) based on their secondary structure and splicing mechanism (Saldanha et al. 1993), with group I introns being abundant in fungal mitogenomes. Different fungal species or even different individuals of a particular fungus may show diversity in number and insertion position of mitochondrial introns (Kosa et al. 2006; Zhang et al. 2015; Zhang et al. 2017a;

(c) The Author(s). 2019 Open Access This article is distributed under the terms of the Creative Commons Attribution 4.0 International License (http://creativecommons.org/licenses/by/4.0/), which permits unrestricted use, distribution, and 
Wang et al. 2018; Fan et al. 2019; Nie et al. 2019). Introns contribute to fungal mitogenome expansion/variability and represent an ideal marker for understanding fungal evolution (Zhang et al. 2015).

Currently, there has been a nomenclature for introns present in rRNA genes (Johansen and Haugen 2001). According to the nomenclature, introns are often found at a limited number of insertion sites in highly conserved regions of rRNA genes from nuclei, mitochondria, and chloroplasts, and therefore, a given rRNA sequence can be aligned with the chosen standard rRNA sequences of Escherichia coli to locate and name potential introns. For mitochondrial protein-coding genes, however, it is difficult to align their sequences with corresponding $E$. coli sequences due to high sequence divergence. In most literatures, introns in protein-coding genes are generally named serially according to their appearance in a particular host gene (e.g., cox1-i1, cox1-i2, and cox1-i3) (Deng et al. 2016; Zhang et al. 2017b; Zhang et al. 2017c). This naming strategy is not convenient for scientific communication and comparison of introns across different mitogenomes. A standard nomenclature of mitochondrial introns is needed to avoid confusion when comparing different fungal mitogenomes.

In our previous studies, we have tried to designate introns based on their insertion positions, but a mitogenome is arbitrarily selected from species under investigation (Fan et al. 2019; Zhang et al. 2019). In this study, we aim to propose a standard nomenclature for introns in protein-coding genes in fungal mitogenomes and test its applicability using fungal species from a broad range of taxonomic classification. To know if the suggested nomenclature can apply to "cross-kingdom" mitochondrial introns, some plant/protist/animal introns are also examined.

\section{METHODS}

In order to establish a standard nomenclature for introns in protein-coding genes across the kingdom Fungi, it is necessary to find an appropriate reference mitogenome. By looking at fungal species with available mitogenomes, we choose the mitogenome of the cyclosporin-producing fungus Tolypocladium inflatum ARSEF 3280 (accession number NC_036382) as the reference mitogenome. The 25,328-bp mitogenome of T. inflatum contains all the 15 protein-coding genes typically found in fungal mitogenomes, and there is no intron in any of these proteincoding genes (Zhang et al. 2017d). We did not choose the best-understood model fungi: 'baker's yeast' Saccharomyces cerevisiae, the fission yeast Schizosaccharomyces pombe, the opportunistic fungal pathogen Candida albicans, the filamentous euascomycete Neurospora crassa, etc. This is because the yeasts $S a$. cerevisiae and Sc. pombe both lack genes coding for NADH dehydrogenases in their mitogenomes (Foury et al. 1998), and C. albicans and $N$. crassa contain introns in many different protein-coding genes (Borkovich et al. 2004; Bartelli et al. 2013). We also did not choose the human mitochondrial genome, which was selected as the reference to name introns found in nad5 and cox1 in certain metazoans (Emblem et al. 2011). This is because the human mitogenome contains only 13 standard protein-coding genes without atp 9 and rps3. The latter two genes are known to harbor introns in fungal mitogenomes.

Both basal and higher fungi may contain introns in their mitogenomes. We randomly selected representative species in each fungal phylum to locate and name possible introns (Table 1). Determination of the insertion position of an intron relies on alignment between sequences of its host gene and corresponding gene sequences of $T$. inflatum (Additional file 1). Although there are many sequence alignment programs available, we recommend using MAFFT (https://mafft.cbrc.jp/alignment/software/), which is fast when aligning long sequences containing many introns and can always generate satisfactory alignment according to our experience. The default setting of MAFFT works well in most cases. If exon-intron boundaries are not correctly identified (probably due to the interference of intron sequences or presence of short exons) under the default settings, one may consider adjusting the alignment parameters (e.g., try 'Unalignlevel $>0$ ' and possibly 'Leave gappy regions' by selecting the G-INS-1 or G-INS-i alignment strategy) and/or importing additional sequences to align from a species closely related the test species. In addition, it is always advisable to refer to known annotation results and/or characteristic nucleotides at splice sites of group I/II introns (Cech 1988) to ensure correct alignment and identification of exon-intron boundaries.

\section{RESULTS AND DISCUSSION}

We propose a new nomenclature system for introns in fungal mitochondrial protein-coding genes based on (1) three-letter abbreviation of host scientific name, (2) host gene name, (3) one capital letter $\mathrm{P}$ (for group I introns, meaning position or primary for easy memorization), $S$ (for group II introns, meaning site or secondary), or U (for introns with unknown types), and (4) intron insertion site in the host gene according to T. inflatum (Additional file 1). When there is no ambiguity (e.g., when just talking about introns in a particular species or in a particular host gene of a species), host scientific name and/or host gene name may be omitted. In any case, however, the letter $\mathrm{P} / \mathrm{S} / \mathrm{U}$ and insertion site of an intron should never be omitted. Using the nomenclature, previously reported introns could be renamed. Examples of renaming are the group II intron Sce.cox1S169 (former aI1) from Saccharomyces cerevisiae cox1 at site 169, and the group I intron Cgl.cox1P240 (former CgCox1.1) from Candida glabrata cox1 at position 


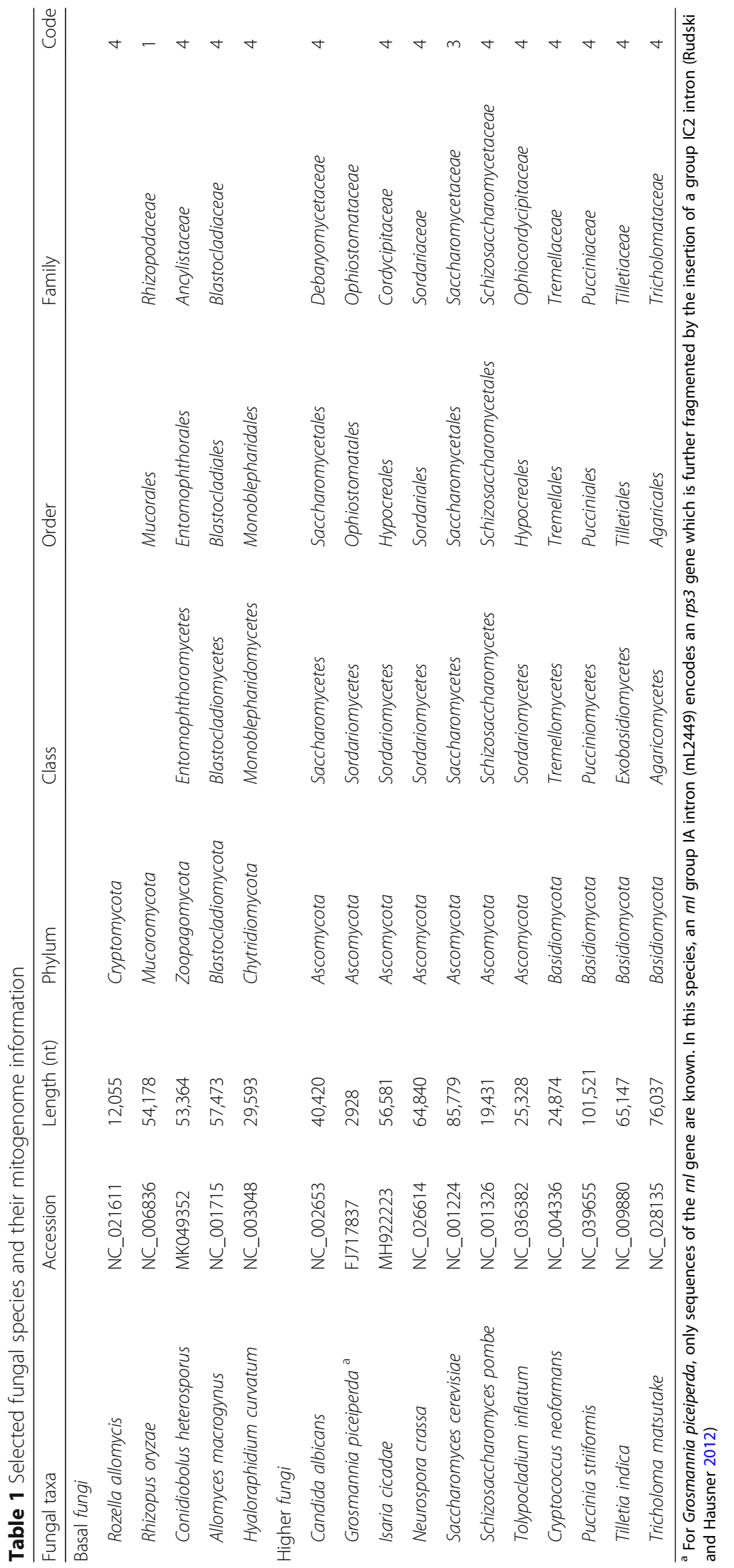


240. Other examples are included in Table 2 (lines 1-10). We hope future studies follow this proposed nomenclature to ensure direct comparison across different studies.

The suggested nomenclature is flexible to fit some special conditions. Firstly, although we suggest three-letter abbreviation of host scientific name, four-or-more-letter abbreviation may be used in cases where the three-letter abbreviation cannot discriminate among all species under investigation. An example is introns at position 717 in nad5 in Candida pseudojiufengensis (Cpse.nad5U717) and Candida psychrophila (Cpsy.nad5P717) (Table 2, lines 11-12). Secondly, twintrons (twin introns) have been described from some fungal mitogenomes with various combinations of group I or II introns nested inside each other or situated next to each other (Hafez and Hausner 2015; Deng et al. 2016). The internal/external or upstream/ downstream members of a twintron could be named alphabetically. An example is the side-by-side twintron in cox3 in Hypomyces aurantius, where two group IA introns are arranged in tandem (Deng et al. 2016). The upstream intron of the twintron can be named as Hau.cox3P640a and the downstream one as Hau.cox3P640b (Table 2, lines 13-14). Finally, although introns present at an identical insertion site among different strains of a particular species are generally conserved, distantly related introns are sometimes detected among different strains. Introns of this kind can be named numerically. For example, Hth.cobP429 in different strains of Hirsutella thompsonii showed length variations (e.g., $2.7 \mathrm{~kb}$ in ARSEF 9457 and $4.8 \mathrm{~kb}$ in ARSEF 1947) (Wang et al. 2018), and the two variants may be named as Hth.cobP429-1 in ARSEF 9457 and Hth.cobP429-2 in ARSEF 1947 (Table 2, lines 15-16).

The suggested nomenclature has been successfully applied to name introns in 16 fungi from different phyla, including both basal and higher fungal lineages (Table 3). These fungi contain introns in all proteincoding genes except $a t p 8, n a d 2$, and $n a d 6$, and $c o b$ and cox 1 are most frequently invaded by introns. These introns are mostly group I introns, but we also find few group II introns as well as few introns with undetermined types. There are a total of 149 introns at 74 insertion sites in these fungi. Using the suggested nomenclature, intron positions in a particular gene can be directly observed and compared across different species. We find some points frequently inserted by introns in different species (e.g., cobP490, cox1P386, cox1P720, cox1P1107). From the intron insertion site numbers, one can also easily understand the phase of

Table 2 Representative examples of the new nomenclature of introns in protein-coding genes a

\begin{tabular}{|c|c|c|c|c|c|c|c|}
\hline Line & New name & Old name & Fungal taxa & Host gene & Accession & Note & Reference \\
\hline 1 & Sce.cox1S169 & al1 & Saccharomyces cerevisiae & $\cos 1$ & NC_001224 & Group II intron & Foury et al. 1998 \\
\hline 2 & Sce.cox1P971 & $\mathrm{al} 5 \mathrm{a}$ & Saccharomyces cerevisiae & $\operatorname{cox} 1$ & NC_001224 & Group I intron & Foury et al. 1998 \\
\hline 3 & Sce.cox1P1107 & al5 $\beta$ & Saccharomyces cerevisiae & $\operatorname{cox} 1$ & NC_001224 & Group I intron & Foury et al. 1998 \\
\hline 4 & Sce.cox1S1132 & al5y & Saccharomyces cerevisiae & $\operatorname{cox} 1$ & NC_001224 & Group II intron & Foury et al. 1998 \\
\hline 5 & Cgl.cox1P240 & CgCox1.1 & Candida glabrata & $\operatorname{cox} 1$ & NC_004691 & Group I intron & Koszul et al. 2003 \\
\hline 6 & Cgl.cox1P386 & CgCox 1.2 & Candida glabrata & $\operatorname{cox} 1$ & NC_004691 & Group I intron & Koszul et al. 2003 \\
\hline 7 & Cgl.cox1P971 & CgCox1.3 & Candida glabrata & $\operatorname{cox} 1$ & NC_004691 & Group I intron & Koszul et al. 2003 \\
\hline 8 & Cme.cobP393 & bl1 & Candida metapsilosis & $c o b$ & NC_006971 & Group I intron & Kosa et al. 2006 \\
\hline 9 & Hth.nad1P636 & nad1-i1 & Hirsutella thompsonii & nadl & NC_040165 & Group I intron & Wang et al. 2018 \\
\hline 10 & Ici.atp9P181 & atp9-i1 & Isaria cicadae & $\operatorname{atp} 9$ & MH922223 & Group I intron & Fan et al. 2019 \\
\hline 11 & Cpse.nad5U717 & & Candida pseudojiufengensis & nad5 & NC_022156 & Unknown intron type & Unpublished \\
\hline 12 & Cpsy.nad5P717 & & Candida psychrophila & nad5 & NC_036103 & Group I intron & Unpublished \\
\hline 13 & Hau.cox3P640a & $\cos 3-\mathrm{i} 2$ & Hypomyces aurantius & $\operatorname{cox} 3$ & NC_030206 & 1st one in twintron & Deng et al. 2016 \\
\hline 14 & Hau.cox3P640b & $\cos 3-i 2$ & Hypomyces aurantius & $\operatorname{cox} 3$ & NC_030206 & 2nd one in twintron & Deng et al. 2016 \\
\hline 15 & Hth.cobP429-1 & cob-i2 & Hirsutella thompsonii & $c o b$ & NC_040165 & Strain: ARSEF 9457 & Wang et al. 2018 \\
\hline 16 & Hth.cobP429-2 & cob-i2 & Hirsutella thompsonii & $c o b$ & МH367296 & Strain: ARSEF 1947 & Wang et al. 2018 \\
\hline 17 & Zsa.nad5P717 & ND5-717 & Zoanthus sansibaricus & nad5 & KY888672 & Coral: Group I intron & Chi and Johansen 2017 \\
\hline 18 & Zsa.cox1P867 & COl-867 & Zoanthus sansibaricus & $\operatorname{cox} 1$ & KY888672 & Coral: Group I intron & Chi and Johansen 2017 \\
\hline 19 & Mbr.nad5P717 & & Monosiga brevicollis & nad5 & AF538053 & Protist: Group I intron & Burger et al. 2003 \\
\hline 20 & Ddi.cox2P357 & & Dictyostelium discoideum & $\cos 1 / 2$ & NC_000895 & Protist: Group I intron & Ogawa et al. 2000 \\
\hline 21 & Mpo.nad5P717 & & Marchantia polymorpha & nad5 & M68929 & Plant: Group I intron & Oda et al. 1992 \\
\hline 22 & Ath.cox2S691 & & Arabidopsis thaliana & $\cos 2$ & NC_037304 & Plant: Group II intron & Sloan et al. 2018 \\
\hline
\end{tabular}

a Examples from lines 1 to 16 are fungal species, and those from lines 17 to 22 are plant/protist/animal species as indicated in the column "Note" 
Zhang and Zhang IMA Fungus

(2019) 10:15

Page 5 of 8

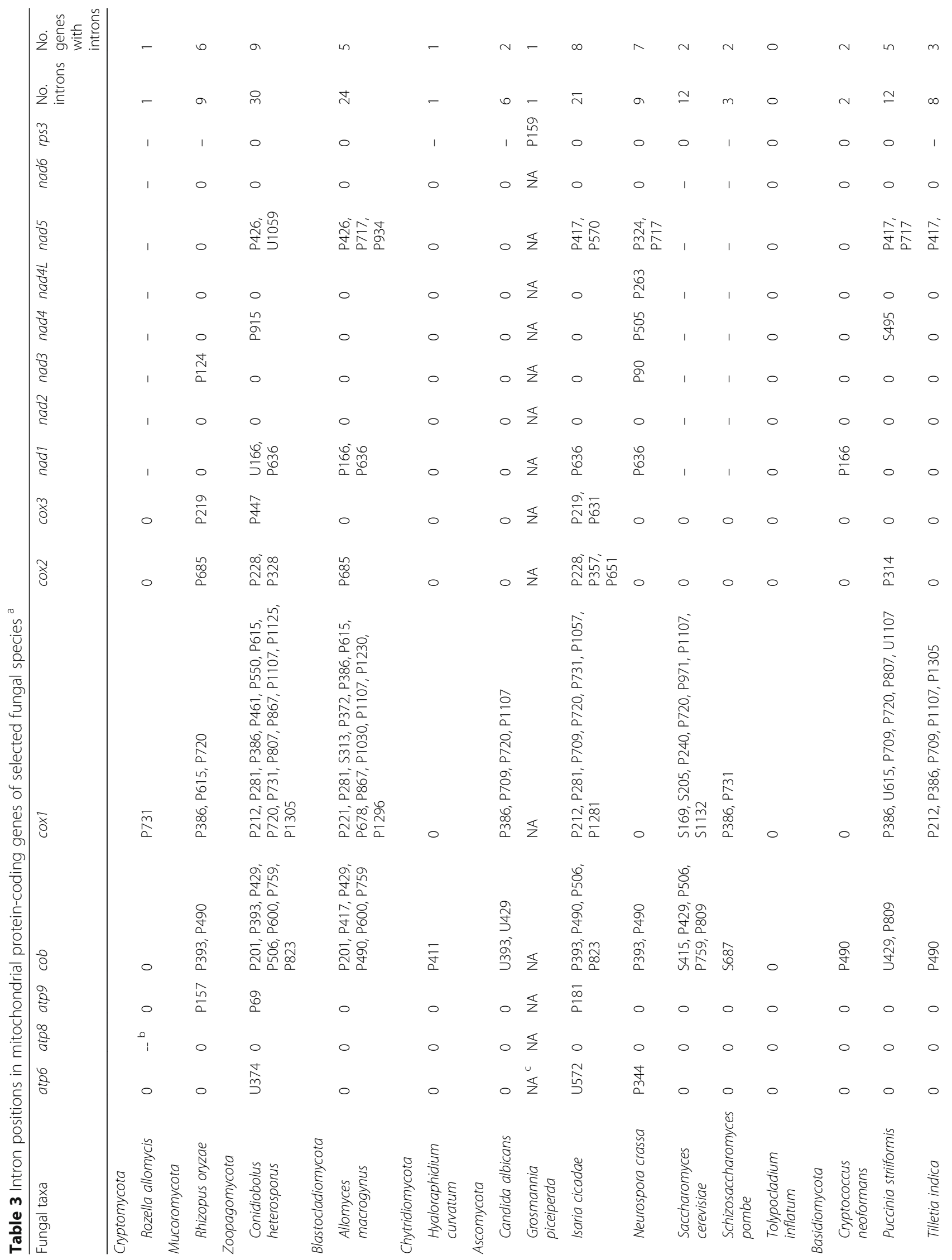


Zhang and Zhang IMA Fungus ～～（2019) 10:15

Page 6 of 8

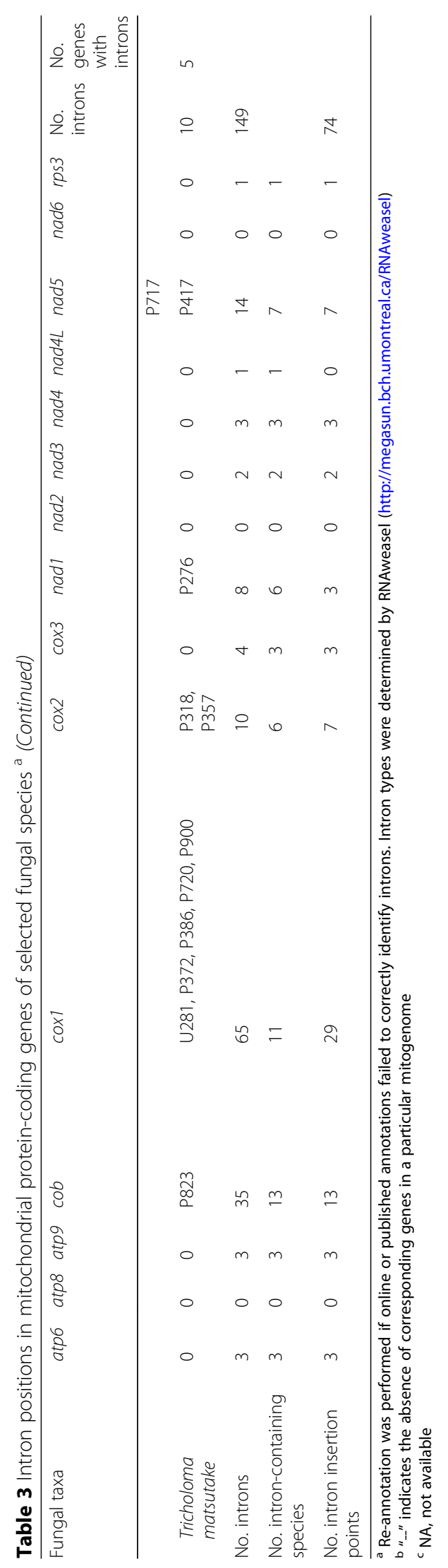


an intron, which is phase 0 when an intron inserts between two codons (e.g., cobP393), and phase 1 or 2 when an intron inserts within a codon (e.g., cox1S205, cox1P386). These introns are often found at highly conserved regions (Additional file 2).

In addition to fungi, plants and protists (but rarely in animals) also contain group I or II introns in their mitochondrial genes (Oda et al. 1992; Ogawa et al. 2000; Burger et al. 2003; Chi and Johansen 2017). The nomenclature suggested in this study could potentially apply to plant/protist/ animal mitochondrial introns (Table 2, lines 17-22; Additional file 2). Plant mitogenomes, however, are also known to encode several intron-containing protein genes (e.g., nad7, ccmC, rps10, rpl2) that are absent in fungal mitogenomes (Zhang et al. 2011; Sloan et al. 2018). Introns are even found in tRNA-coding genes in plant mitogenomes (Smith et al. 2011). An additional plant reference is necessary to name introns unique to plant mitogenomes.

\section{CONCLUSIONS}

A standard nomenclature was suggested for introns in protein-coding genes in fungal mitogenomes. It was proved feasible by naming introns present in mitogenomes of 16 fungi from a broad range of taxonomic classification, and it also had the potential to name introns in plant/protist/animal mitogenomes. Future studies should follow the proposed nomenclature to ensure direct comparison across different studies.

\section{Additional files}

Additional file 1: Sequences of protein-coding genes of Tolypocladium inflatum ARSEF 3280 (accession number NC 036382). Insertion site of group I introns are shown in red, group || introns in green, and introns with undetermined intron types in shade. (DOCX $21 \mathrm{~kb}$ )

Additional file 2: Intron insertion sites for 22 common introns. Exon sequences of cob, cox1, cox2, nad1, and nad5 of different fungal taxa plus few non-fungal taxa were aligned by MAFFT, and visualization of the aligned sequences was performed using ESPript 3.0 (Robert and Gouet 2014) under default settings. Refer to Tables 1 and 2 for organisms represented by accession numbers, and the accession numbers of non-fungal taxa are marked in red boxes. Insertion sites of introns are shown using upward arrows. For phase 0 introns, conserved amino acids before and after insertion sites are listed. The amino acid glycine (G) is frequently seen before insertion sites of phase 0 introns. For phase 1 or 2 introns, conserved amino acids at insertion sites are given, and corresponding triplet codons are marked by a horizontal line. (PPTX $2235 \mathrm{~kb}$ )

\section{Acknowledgements}

Authors are thankful to the editor and two anonymous reviewers for their suggestions that helped us improve the manuscript.

\section{Adherence to national and international regulations} Not applicable.

\section{Authors' contributions}

YJZ designed the research and wrote the manuscript. SZ performed the research. Both authors read and approved the final manuscript.

\section{Funding}

This study was funded by the National Natural Science Foundation of China (31872162), the Research Project Supported by Shanxi Scholarship Council of China (2017-015), Hundred Talents Program of Shanxi Province, and the Special Fund for Large Scientific Instruments and Equipment in Shanxi Province.

Availability of data and materials

All data used in this study are publicly available.

Ethics approval and consent to participate

Not applicable.

\section{Consent for publication}

Not applicable.

\section{Competing interests}

The authors declare that they have no competing interests.

\section{Author details}

${ }^{1}$ Institute of Applied Chemistry, Shanxi University, Taiyuan 030006, China. ${ }^{2}$ School of Life Science, Shanxi University, Taiyuan 030006, China.

Received: 8 June 2019 Accepted: 20 August 2019

Published online: 10 October 2019

\section{References}

Bartelli TF, Ferreira RC, Colombo AL, Briones MRS (2013) Intraspecific comparative genomics of Candida albicans mitochondria reveals non-coding regions under neutral evolution. Infection, Genetics and Evolution 14:302-312

Borkovich KA, Alex LA, Yarden O, Freitag M, Turner GE, Read ND et al (2004) Lessons from the genome sequence of Neurospora crassa: tracing the path from genomic blueprint to multicellular organism. Microbiology and Molecular Biology Reviews 68:1-108

Bullerwell CE, Lang BF (2005) Fungal evolution: the case of the vanishing mitochondrion. Current Opinion in Microbiology 8:362-369

Burger G, Forget L, Zhu Y, Gray MW, Lang BF (2003) Unique mitochondrial genome architecture in unicellular relatives of animals. Proceedings of the National Academy of Sciences 100:892-897

Cannon P, Aguirre-Hudson B, Aime MC, Ainsworth AM, Bidartondo MI, Gaya E et al (2018) Definition and diversity. In: Willis KJ (ed) State of the World's Fungi report. Royal Botanic Gardens, Kew, pp 4-11

Cech TR (1988) Conserved sequences and structures of group I introns: building an active site for RNA catalysis--a review. Gene 73:259-271

Chi SI, Johansen SD (2017) Zoantharian mitochondrial genomes contain unique complex group I introns and highly conserved intergenic regions. Gene 628:24-31

Deng Y, Zhang Q, Ming R, Lin L, Lin X, Lin Y et al (2016) Analysis of the mitochondrial genome in Hypomyces aurantius reveals a novel twintron complex in fungi. International Journal of Molecular Sciences 17:1049

Emblem A, Karlsen BO, Evertsen J, Johansen SD (2011) Mitogenome rearrangement in the cold-water scleractinian coral Lophelia pertusa (Cnidaria, Anthozoa) involves a long-term evolving group I intron. Molecular Phylogenetics and Evolution 61:495-503

Fan W-W, Zhang S, Zhang Y-J (2019) The complete mitochondrial genome of the Chan-hua fungus Isaria cicadae: a tale of intron evolution in Cordycipitaceae. Environmental Microbiology 21:864-879

Foury F, Roganti T, Lecrenier N, Purnelle B (1998) The complete sequence of the mitochondrial genome of Saccharomyces cerevisiae. FEBS Letters 440:325-331

Hafez M, Hausner G (2015) Convergent evolution of twintron-like configurations: one is never enough. RNA Biology 12:1275-1288

Hawksworth DL, Lücking R (2017) Fungal diversity revisited: 2.2 to 3.8 million species. Microbiol Spectrum 5: FUNK-0052-2016

Johansen S, Haugen P (2001) A new nomenclature of group I introns in ribosomal DNA. RNA 7:935-936

Kosa P, Valach M, Tomaska L, Wolfe KH, Nosek J (2006) Complete DNA sequences of the mitochondrial genomes of the pathogenic yeasts Candida orthopsilosis and Candida metapsilosis: insight into the evolution of linear DNA genomes from mitochondrial telomere mutants. Nucleic Acids Research 34:2472-2481

Koszul R, Malpertuy A, Frangeul L, Bouchier C, Wincker P, Thierry A et al (2003) The complete mitochondrial genome sequence of the pathogenic yeast Candida (Torulopsis) glabrata. FEBS Letters 534:39-48 
Lang BF (2018) Mitochondrial genomes in Fungi. In: Wells RD, Bond JS, Klinman J, Masters BSS (eds) Molecular Life Sciences. Springer, New York, pp 722-728

Nie Y, Wang L, Cai Y, Tao W, Zhang Y-J, Huang B (2019) Mitochondrial genome of the entomophthoroid fungus Conidiobolus heterosporus provides insights into evolution of basal fungi. Applied Microbiology and Biotechnology 103: 1379-1391

Oda K, Yamato K, Ohta E, Nakamura Y, Takemura M, Nozato N et al (1992) Gene organization deduced from the complete sequence of liverwort Marchantia polymorpha mitochondrial DNA. A primitive form of plant mitochondrial genome. Journal of Molecular Biology 223:1-7

Ogawa S, Yoshino R, Angata K, Iwamoto M, Pi M, Kuroe K et al (2000) The mitochondrial DNA of Dictyostelium discoideum: complete sequence, gene content and genome organization. Molecular \& General Genetics 263:514-519

Robert X, Gouet P (2014) Deciphering key features in protein structures with the new ENDscript server. Nucleic Acids Research 42:W320-W324

Rudski SM, Hausner G (2012) The mtDNA rps3 locus has been invaded by a group I intron in some species of Grosmannia. Mycoscience 53:471-475

Saldanha R, Mohr G, Belfort M, Lambowitz AM (1993) Group I and group II introns. The FASEB Journal 7:15-24

Sloan DB, Wu Z, Sharbrough J (2018) Correction of persistent errors in Arabidopsis reference mitochondrial genomes. Plant Cell 30:525-527

Smith DR, Burki F, Yamada T, Grimwood J, Grigoriev IV, Van Etten JL, Keeling PJ (2011) The GC-rich mitochondrial and plastid genomes of the green alga Coccomyxa give insight into the evolution of organelle DNA nucleotide landscape. PLoS One 6:e23624

Spatafora JW, Aime MC, Grigoriev IV, Martin F, Stajich JE, Blackwell M (2017) The fungal tree of life: from molecular systematics to genome-scale phylogenies. Microbiology Spectrum 5: FUNK-0053-2016

van der Giezen M, Tovar J, Clark CG (2005) Mitochondrion-derived organelles in protists and fungi. Int Rev Cytol. 244:175-225

Wang L, Zhang S, Li J-H, Zhang Y-J (2018) Mitochondrial genome, comparative analysis and evolutionary insights into the entomopathogenic fungus Hirsutella thompsonii. Environmental Microbiology 20:3393-3405

Zhang S, Hao AJ, Zhao YX, Zhang XY, Zhang Y-J (2017a) Comparative mitochondrial genomics toward exploring molecular markers in the medicinal fungus Cordyceps militaris. Scientific Reports 7:40219

Zhang S, Wang X-N, Zhang X-L, Liu X-Z, Zhang Y-J (2017b) Complete mitochondrial genome of the endophytic fungus Pestalotiopsis fici: features and evolution. Applied Microbiology and Biotechnology 101:1593-1604

Zhang S, Zhang Y-J, Li Z (2019) Complete mitogenome of the entomopathogenic fungus Sporothrix insectorum RCEF 264 and comparative mitogenomics in Ophiostomatales. Applied Microbiology and Biotechnology 13:5797-5809

Zhang X, Zhang R, Hou S-Y, Shi J, Guo S-D (2011) Research progress on mitochondrial genome of higher plant. Journal of Agricultural Science and Technology 13:23-31

Zhang Y-J, Yang X-Q, Zhang S, Humber RA, Xu J (2017d) Genomic analyses reveal low mitochondrial and high nuclear diversity in the cyclosporinproducing fungus Tolypocladium inflatum. Applied Microbiology and Biotechnology 101:8517-8531

Zhang Y-J, Zhang H-Y, Liu X-Z, Zhang S (2017c) Mitochondrial genome of the nematode endoparasitic fungus Hirsutella vermicola reveals a high level of synteny in the family Ophiocordycipitaceae. Applied Microbiology and Biotechnology 101:3295-3304

Zhang Y-J, Zhang S, Zhang G, Liu X, Wang C, Xu J (2015) Comparison of mitochondrial genomes provides insights into intron dynamics and evolution in the caterpillar fungus Cordyceps militaris. Fungal Genetics and Biology 77:95-107

\section{Publisher's Note}

Springer Nature remains neutral with regard to jurisdictional claims in published maps and institutional affiliations.

Ready to submit your research? Choose BMC and benefit from:

- fast, convenient online submission

- thorough peer review by experienced researchers in your field

- rapid publication on acceptance

- support for research data, including large and complex data types

- gold Open Access which fosters wider collaboration and increased citations

- maximum visibility for your research: over $100 \mathrm{M}$ website views per year

At BMC, research is always in progress.

Learn more biomedcentral.com/submissions 